\title{
Expanded Endoscopic Endonasal Treatment of Primary Intracranial Tumors within the Paranasal Sinuses
}

\author{
Zarina S. Ali, ${ }^{1}$ Shih-Shan Lang, ${ }^{1}$ Nithin D. Adappa, ${ }^{2}$ Ariana Barkley, \\ James N. Palmer, ${ }^{2}$ and John Y. K. Lee ${ }^{1}$ \\ ${ }^{1}$ Department of Neurosurgery, University of Pennsylvania, Philadelphia, PA 19104, USA \\ ${ }^{2}$ Department of Otorhinolaryngology, Head and Neck Surgery, University of Pennsylvania, Philadelphia, PA 19104, USA
}

Correspondence should be addressed to Zarina S. Ali; zarinasali@gmail.com

Received 10 June 2013; Accepted 21 July 2013

Academic Editors: S. Albu, S. Elwany, Z. Habib, Y. Izci, and P. Spennato

Copyright (C) 2013 Zarina S. Ali et al. This is an open access article distributed under the Creative Commons Attribution License, which permits unrestricted use, distribution, and reproduction in any medium, provided the original work is properly cited.

\begin{abstract}
Objective. Meningiomas and schwannomas represent a subset of primary intracranial tumors that are rarely identified exclusively in the paranasal sinuses. Here, we describe our experience with minimally invasive endoscopic endonasal approaches for the treatment of these tumors. Methods. We retrospectively reviewed the clinical, surgical, and radiographic characteristics of adults with pathologically confirmed sinonasal meningiomas and schwannomas located within the paranasal sinuses that were resected via an expanded endoscopic endonasal approach. Results. Five patients (1 male, 4 females) underwent an endoscopic endonasal approach for resection of sinonasal tumor. Clinical symptomatology most commonly included nasal obstruction, in addition to headache, jaw pain, anosmia, and chronic rhinosinusitis. Tumors were located exclusively within the sinonasal cavity and were on average $2.2 \mathrm{~cm}$ (range 1.4-3.8 cm). Pathology revealed 2 cases of meningioma and 3 cases of schwannoma. No evidence of tumor recurrence occurred over average followup of 1.5 years (range 0.11-3.9 years). Conclusion. Our case series suggests that an expanded endoscopic endonasal approach with a combined neurosurgical-otorhinolaryngologic team for the resection of sinonasal meningiomas and schwannomas offers an effective treatment option. Further studies that include a larger number of patients over a longer follow-up period are required to compare outcomes between minimally invasive and open approaches.
\end{abstract}

\section{Introduction}

Sinonasal schwannomas and meningiomas represent a rare subset of benign sinonasal tumors. Schwannomas originate from neural the crest-derived Schwann cells that line central nerve sheaths. In the head and neck, these tumors are located less than $4 \%$ of the time in the sinonasal cavity [1]. In contrast, meningiomas arise from arachnoid cap cells and are even less commonly associated with an extracranial origin $(<2 \%)$, of which the sinonasal tract is most prevalent [2].

Traditional, transcranial approaches to these lesions are inherently morbid, due to significant brain retraction and complications associated with skin incision and craniotomy. Endoscopic endonasal approaches are becoming increasingly utilized to access these skull base pathologies. For these small, benign lesions extending into the paranasal sinuses, endoscopic approaches offer a minimally invasive approach to tumor resection and, in some cases, complete tumor resection. Endoscopic techniques provide a panoramic view of the lesion and associated anatomy not seen with the microscopic approach. In addition, advances in skull base reconstruction have allowed a significant reduction in postoperative cerebrospinal fluid leak with transnasal endoscopic approaches. There are limited case series and reports describing the clinical presentation and surgical management of sinonasal schwannomas and meningiomas. Here, we report our experience of a combined neurosurgical-otolaryngologic approach for these rare lesions.

\section{Methods}

2.1. Study Design and Subjects. We retrospectively reviewed a database of all patients who underwent endoscopic endonasal approaches for the treatment of sinonasal schwannomas and meningiomas without a combined open surgical approach over the last decade (2002-2012). A retrospective analysis of 
TABle 1: Patient characteristics.

\begin{tabular}{lcccccccc}
\hline Case no. & Date & Sex & Age (years) & Presenting symptoms & Site & Size (cm) & Pathology & Followup \\
\hline 1 & 2002 & Male & 26 & Nasal obstruction & Left ethmoid, osteomeatal & 3.8 & Schwannoma & 1.6 \\
2 & 2006 & Female & 35 & Headache & Left pterygopalatine fossa & 1.4 & Schwannoma & 0.14 \\
3 & 2012 & Female & 45 & Left upper jaw pain & Left foramen rotundum & 1.7 & Meningioma & 0.11 \\
4 & 2008 & Female & 55 & Nasal obstruction, anosmia & Right cribiform plate & 2.8 & Schwannoma & 1.8 \\
5 & 2008 & Female & 59 & Chronic rhinosinusitis & Right nasal cavity & 1.4 & Meningioma & 3.9 \\
\hline
\end{tabular}

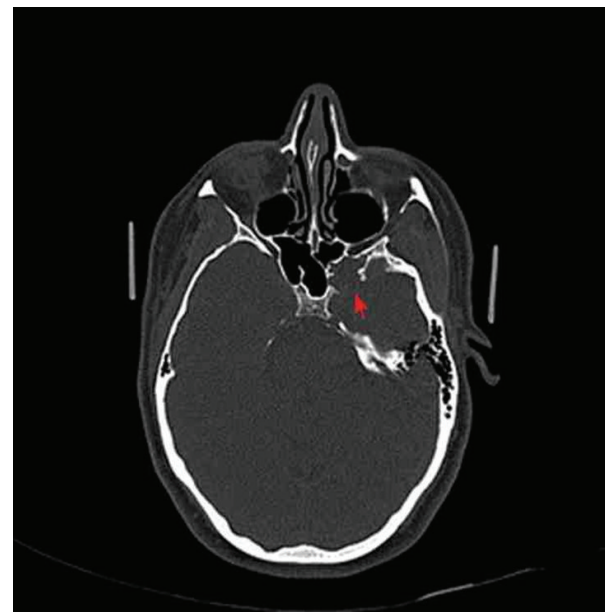

(a)

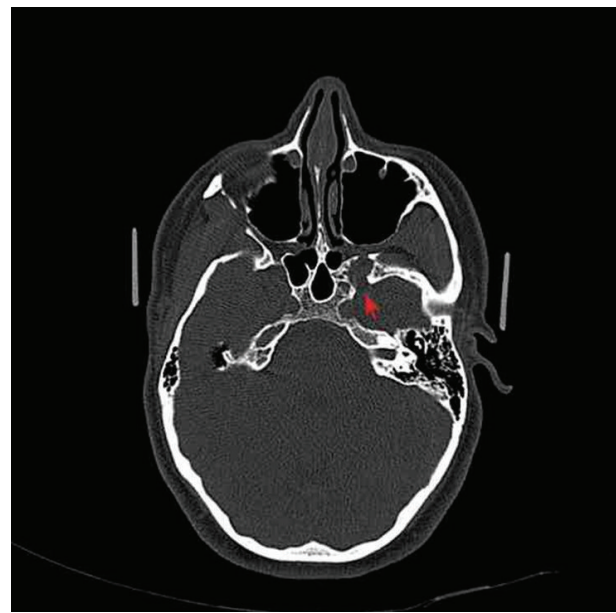

(b)

Figure 1: Preoperative unenhanced maxillofacial CT. (a) Axial cut, bone window, demonstrates an expansile lytic lesion centered in the left sphenoid bone (arrow) and shows expansion of the left foramen rotundum (b).

these patients was performed with approval from the university institutional review board.

2.2. Clinical Data. Patient data, including demographic information, clinical presentation, radiologic films and reports, management decision analysis, and follow-up information, was abstracted from inpatient hospital records and outpatient clinic charts.

\section{Results}

From 2002 to 2012, five patients presented to the neurosurgical or otorhinolaryngology clinic with radiographic evidence of a sinonasal tumor. Table 1 summarizes the patient demographics. $80 \%$ of patients were female $(n=4)$ and only $20 \%$ were male $(n=1)$. Median age at the time of surgery was 45 years (range from 26 to 59 years). The most common clinical presentation was nasal obstruction (40\%). Other presenting symptoms included headache, jaw pain, anosmia, and chronic rhinosinusitis. No patient had a medical history of neurofibromatosis 2, a disorder in which meningiomas and schwannomas are commonly associated. The mean tumor size was $2.2 \mathrm{~cm}$ (range $1.4-3.8 \mathrm{~cm}$ ). Tumor locations included the nasal cavity as well as ethmoid and sphenoid sinuses, cribriform plate, pterygopalatine fossa, and osteomeatal complex. All patients underwent transnasal endoscopic approaches by the neurosurgical-otorhinolaryngologic team. Pathology was confirmed and routine immunohistochemical stains revealed meningioma in $40 \%$ of cases $(n=2)$ and schwannoma in $60 \%$ of cases $(n=3)$. Tumor recurrence did not occur during the study period. The mean followup was 1.5 yrs (range 0.11-3.9 years).

3.1. Case Example. Case 3 is a 45 -year-old female who presented with a two-month history of left ear, jaw, and eye in the setting of presumed chronic rhinosinusitis. Her jaw pain was noted to increase with sneezing and other Valsalva maneuvers. She had no history of epistaxis, anosmia, or headache. Nasal endoscopic examination was within normal limits. Computed tomography (CT) showed an abnormal soft tissue dense mass with bony destruction of the left skull base, adjacent to the sphenoid sinus with expansion of the left foramen rotundum (Figure 1). Magnetic resonance imaging (MRI) revealed a $17 \mathrm{~mm}$ well-defined, enhancing mass in the lateral inferior left sphenoid sinus with involvement of the foramen rotundum and minimal expansion of the anterior inferior aspect of the left cavernous sinus (Figure 2). Differential diagnosis favored trigeminal schwannoma but included chondrosarcoma, cystic fibrous dysplasia, and plasmacytoma.

The patient underwent resection of the lesion via an extended transnasal endoscopic approach with the neurosurgical-otolaryngologic teams using an intraoperative frameless stereotactic computer-assisted surgical navigation system (see Supplemental Video available online at http://dx.doi.org/ $10.1155 / 2013 / 129780)$. Approach to the skull base tumor was 


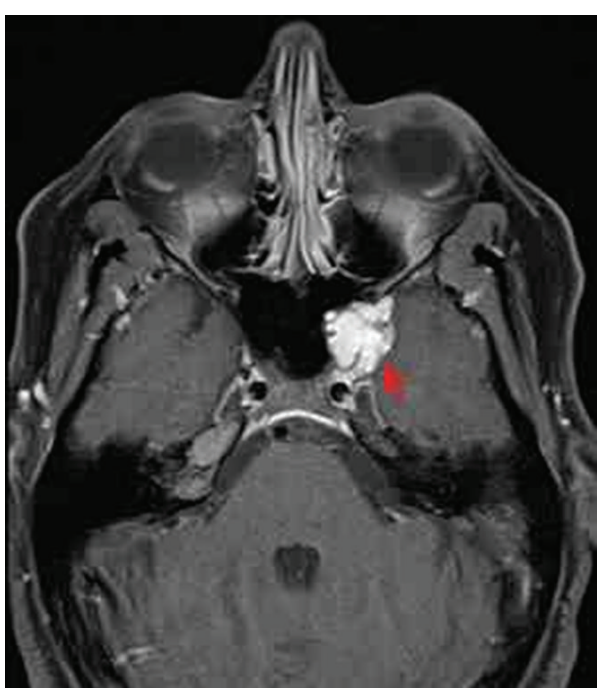

(a)

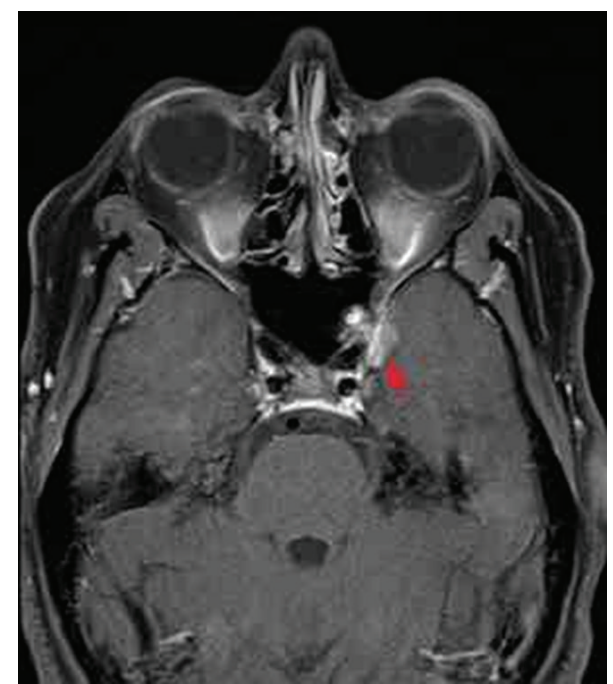

(b)

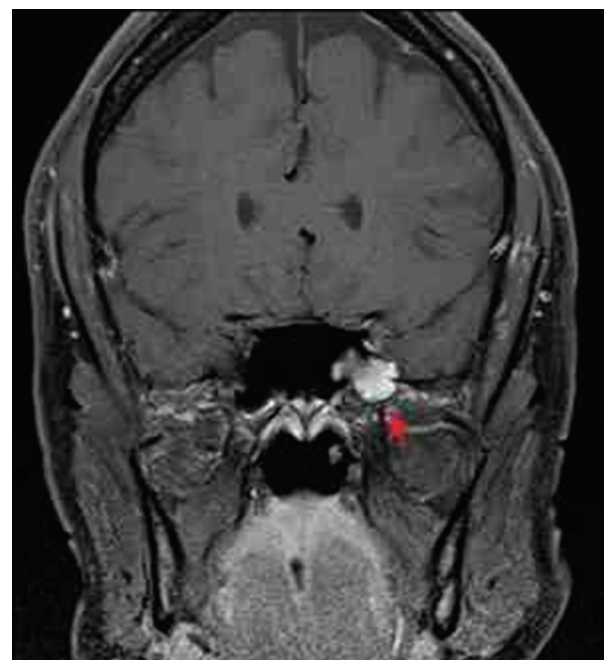

(c)

FIgURE 2: Preoperative enhanced orbital MRI. (a) Axial T1 MRI shows a solidly enhancing lesion (arrow) in the lateral inferior left sphenoid sinus with involvement of the foramen rotundum and the carotid artery in the anterior inferior cavernous sinus (b). (c) Coronal T1 MRI shows the plane between the lesion and the dura of the middle cranial fossa.

undertaken by first performing a left partial middle turbinectomy, followed by a medial maxillectomy and complete sphenoethmoidectomy on the left side. A partial posterior septectomy was also performed to allow binaural access. Subsequently, the entire floor of the sphenoid sinus was drilled, and the sphenopalatine artery was identified and ligated. Upon wide exposure of this area, the vidian nerve was identified, and its canal was unroofed to expose the floor of the lesion. After circumferentially exposing the tumor, dissection and resection of tumor ensued (Figure 3). Grossly, the lesion appeared to have a well-defined capsule containing tissue of soft consistency. The tumor was noted to be arising from foramen rotundum, and cranial nerve V2 was sacrificed in tumor resection at the base of the middle fossa dura (Figure 4). Closure of skull base was performed with a free septal graft. Intraoperative frozen pathology was consistent with a spindle cell neoplasm with myxoid features. Fixed tissue histological examination confirmed a myxoid spindle cell neoplasm consistent with meningioma. Postoperative recovery was uneventful, and the patient reported complete relief of pain with mild left V2 distribution facial numbness at 1.3 months followup.

\section{Discussion}

Schwannomas and meningiomas are benign tumors rarely located in the sinonasal cavity. Most of these tumors are solitary and sporadic, except when associated with neurofibromatosis 2 [3]. Similar to other sinonasal masses, these tumors present with nonspecific symptoms depending on tumor size and location. In our study, nasal obstruction was the most common chief complaint (40\%). The location of these tumors within the sinonasal cavity varies, and in our series includes 


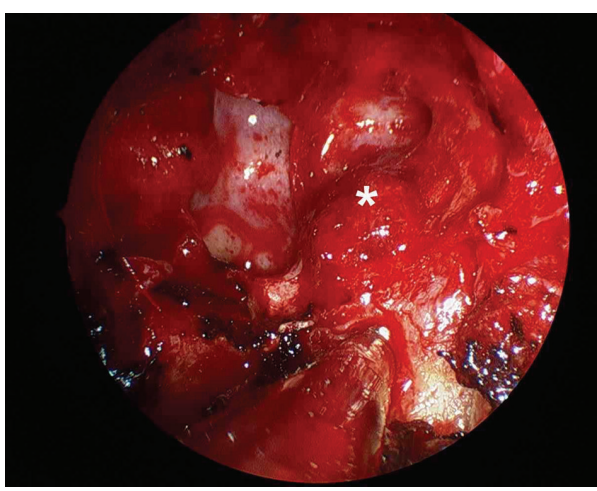

(a)

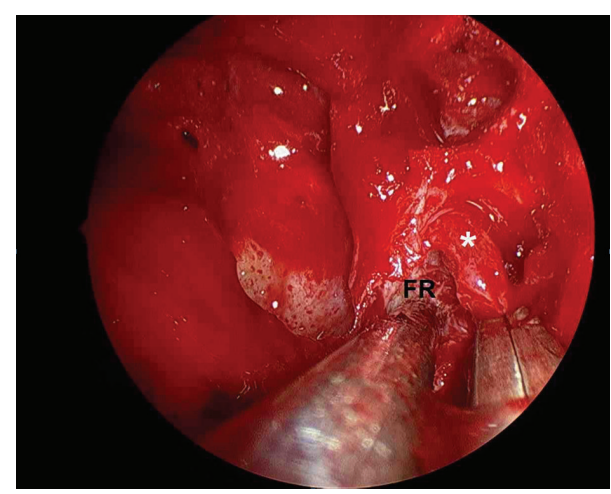

(b)

FIGURE 3: (a) Intraoperative endoscopic view of the lesion $(*)$ in the sphenoid bone. (b) Intraoperative view of the foramen rotundum (FR) and partially resected lesion $(*)$.

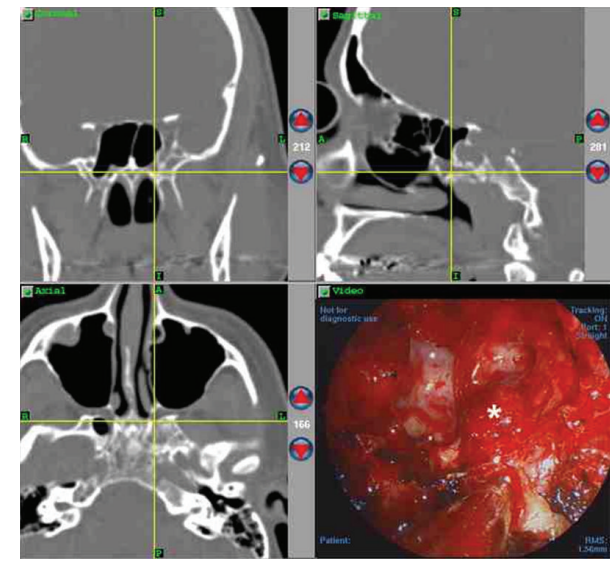

(a)

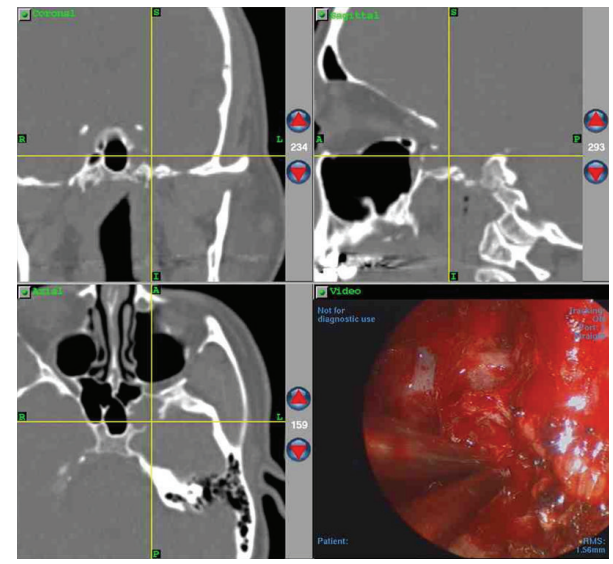

(b)

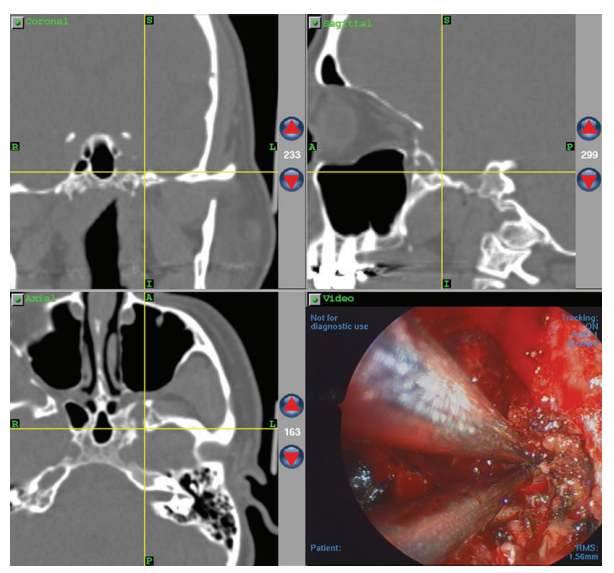

(c)

FIGURE 4: Intraoperative endoscopic view of the lesion with real-time CT-guided navigation. (a) The vidian canal is visualized inferior to the lesion (*). Superior (b) and inferior (c) views of the expanded foramen rotundum are seen on CT.

origins at the ethmoid and sphenoid sinuses, cribriform plate, pterygopalatine fossa, and osteomeatal complex. A recent series by Suh et al. [4] includes a report of seven cases of sinonasal schwannomas located in a similar distribution.
Endoscopic sinus surgery has been used successfully for the resection of other, more common, benign sinonasal masses, including inverted papillomas [5], and malignant lesions [6]. Indeed, in the field of neurosurgery, endoscopic 
approaches have challenged traditional microscopic techniques for treatment of intrasellar tumors [7-10]. Among the advantages of the endoscopic technique is improved visualization of the lesion included, allowing for maximal surgical excision $[11,12]$.

As described previously, the use of the "two-nostrils-fourhands" endoscopic approach by a combined neurosurgicalotolaryngologic team affords significant advantages to the resection of sinonasal schwannomas and meningiomas [13]. In particular, increased maneuverability of the endoscope along with surgical instruments is achieved, which can have dramatic implications for tumor resection in the setting of calcified or fibrous tumor. This approach makes gross total resection via this technique feasible.

In addition to the operative advantages offered by a combined neurosurgical-otolaryngologic approach to these tumors, the potential for skull base involvement and intracranial extension of these tumors warrants neurosurgical care. As in our case example, it is not unusual to identify perineural tumor invasion at the skull base. The benefits of tumor control must be weighed against the morbidities associated with cranial neurectomy by the neurosurgeon. In addition, while none of the cases we describe demonstrated tumor recurrence over the follow-up period, it is important to continue longterm periodic MRI studies on these patients, since tumor recurrence, both locally and intracranially, remains a risk.

This study is inherently limited by its retrospective design, small series of patients, and relatively short-term followup. Further studies are needed to characterize the long-term surgical and clinical outcome of patients treated with this surgical approach.

\section{Conclusion}

Schwannomas and meningiomas represent a benign subset of tumors that rarely originate in the sinonasal cavity. Endonasal endoscopic approaches offer a minimally invasive treatment option for these lesions. Our case series supports the role of the combined neurosurgical-otorhinolaryngologic team in offering the most comprehensive management of these lesions, including complete tumor resection with minimal morbidity.

\section{Conflict of Interests}

None of the authors have any disclosure or conflict of interests.

\section{References}

[1] D. Buob, A. Wacrenier, D. Chevalier et al., "Schwannoma of the sinonasal tract: a clinicopathologic and immunohistochemical study of 5 cases," Archives of Pathology and Laboratory Medicine, vol. 127, no. 9, pp. 1196-1199, 2003.

[2] L. D. R. Thompson and K. A. Gyure, "Extracranial sinonasal tract meningiomas: a clinicopathologic study of 30 cases with a review of the literature," American Journal of Surgical Pathology, vol. 24 , no. 5, pp. 640-650, 2000.
[3] M. S. Dirks, J. A. Butman, H. J. Kim et al., "Long-term natural history of neurofibromatosis Type 2-associated intracranial tumors," Journal of Neurosurgery, vol. 117, no. 1, pp. 109-117, 2012.

[4] J. D. Suh, V. R. Ramakrishnan, P. J. Zhang et al., "Diagnosis and endoscopic management of sinonasal schwannomas," $O R L$, vol. 73, no. 6, pp. 308-312, 2011.

[5] G. Waitz and M. E. Wigand, "Results of endoscopic sinus surgery for the treatment of inverted papillomas," Laryngoscope, vol. 102, no. 8, pp. 917-922, 1992.

[6] P. Nicolai, P. Battaglia, M. Bignami et al., "Endoscopic surgery for malignant tumors of the sinonasal tract and adjacent skull base: a 10-year experience," American Journal of Rhinology, vol. 22, no. 3, pp. 308-316, 2008.

[7] P. Cappabianca, A. Alfieri, and E. De Divitiis, "Endoscopic endonasal transsphenoidal approach to the sella: towards functional endoscopic pituitary surgery (FEPS)," Minimally Invasive Neurosurgery, vol. 41, no. 2, pp. 66-73, 1998.

[8] H.-D. Jho and R. L. Carrau, "Endoscopic endonasal transsphenoidal surgery: experience with 50 patients," Journal of Neurosurgery, vol. 87, no. 1, pp. 44-51, 1997.

[9] G. Zada, D. F. Kelly, P. Cohan, C. Wang, and R. Swerdloff, "Endonasal transsphenoidal approach for pituitary adenomas and other sellar lesions: an assessment of efficacy, safety, and patient impressions," Journal of Neurosurgery, vol. 98, no. 2, pp. 350-358, 2003.

[10] L. E. Bohman, S. C. Stein, J. G. Newman et al., "Endoscopic versus open resection of tuberculum sellae meningiomas: a decision analysis," ORL, vol. 74, no. 5, pp. 255-263, 2012.

[11] C. H. Snyderman, R. L. Carrau, A. B. Kassam et al., "Endoscopic skull base surgery: principles of endonasal oncological surgery," Journal of Surgical Oncology, vol. 97, no. 8, pp. 658-664, 2008.

[12] J. Y. Lee, J. E. Barroeta, J. G. Newman, A. G. Chiu, S. Venneti, and M. S. Grady :, "Endoscopic endonasal resection of anterior skull base meningiomas and mucosa: implications for resection, reconstruction, and recurrence," Journal of Neurological Surgery A, vol. 74, no. 1, pp. 12-17, 2012.

[13] H. R. Briner, D. Simmen, and N. Jones, "Endoscopic sinus surgery: advantages of the bimanual technique," American Journal of Rhinology, vol. 19, no. 3, pp. 269-273, 2005. 


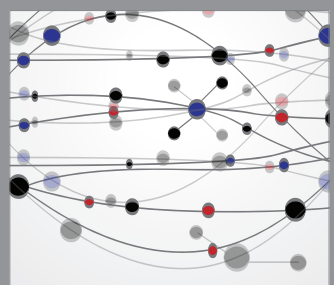

The Scientific World Journal
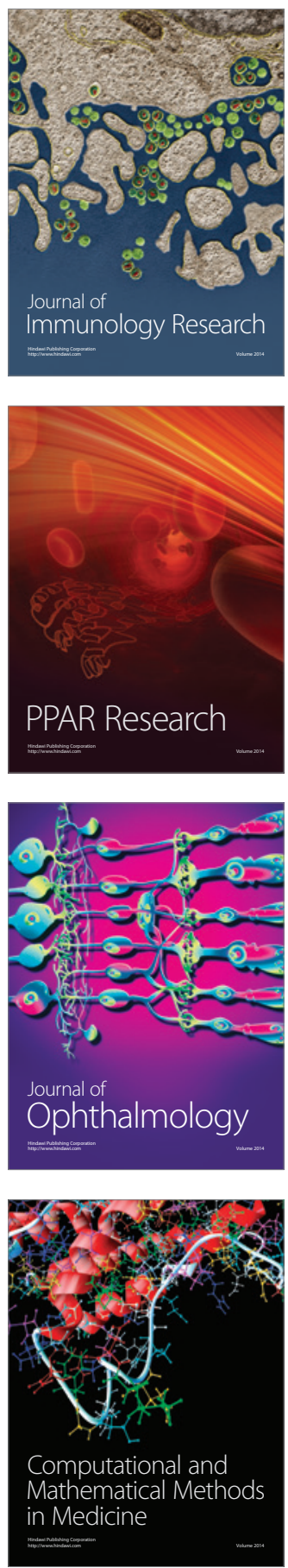

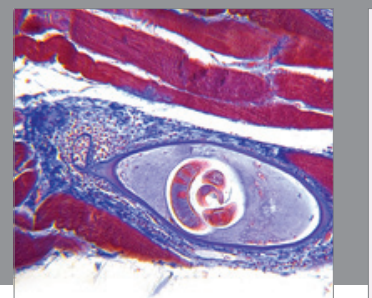

Gastroenterology

Research and Practice
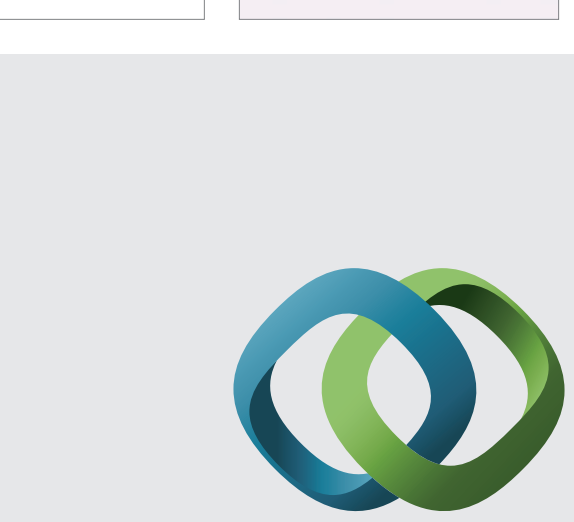

\section{Hindawi}

Submit your manuscripts at

http://www.hindawi.com
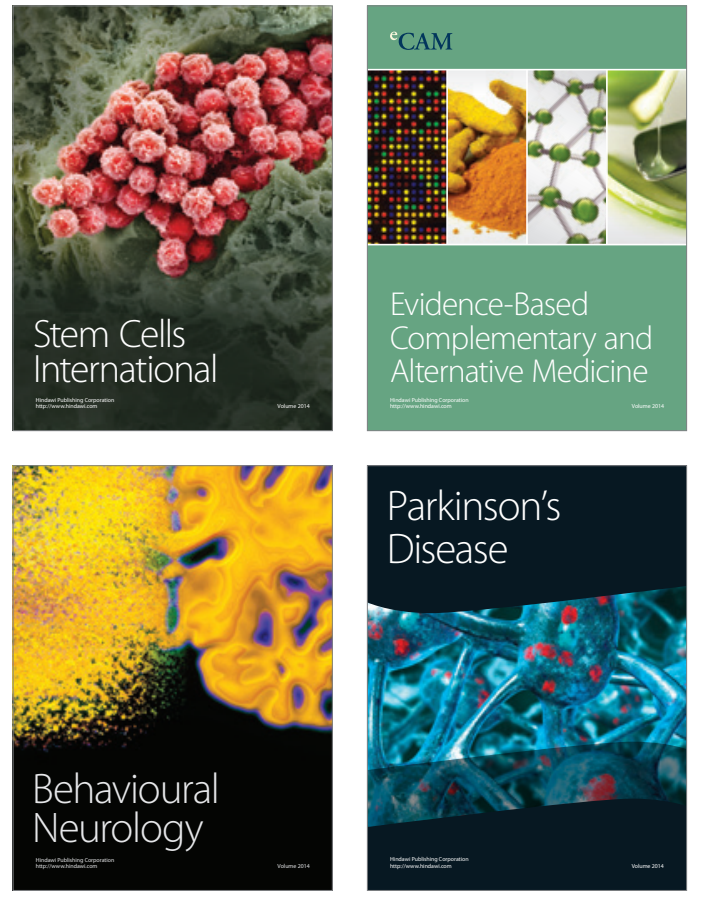
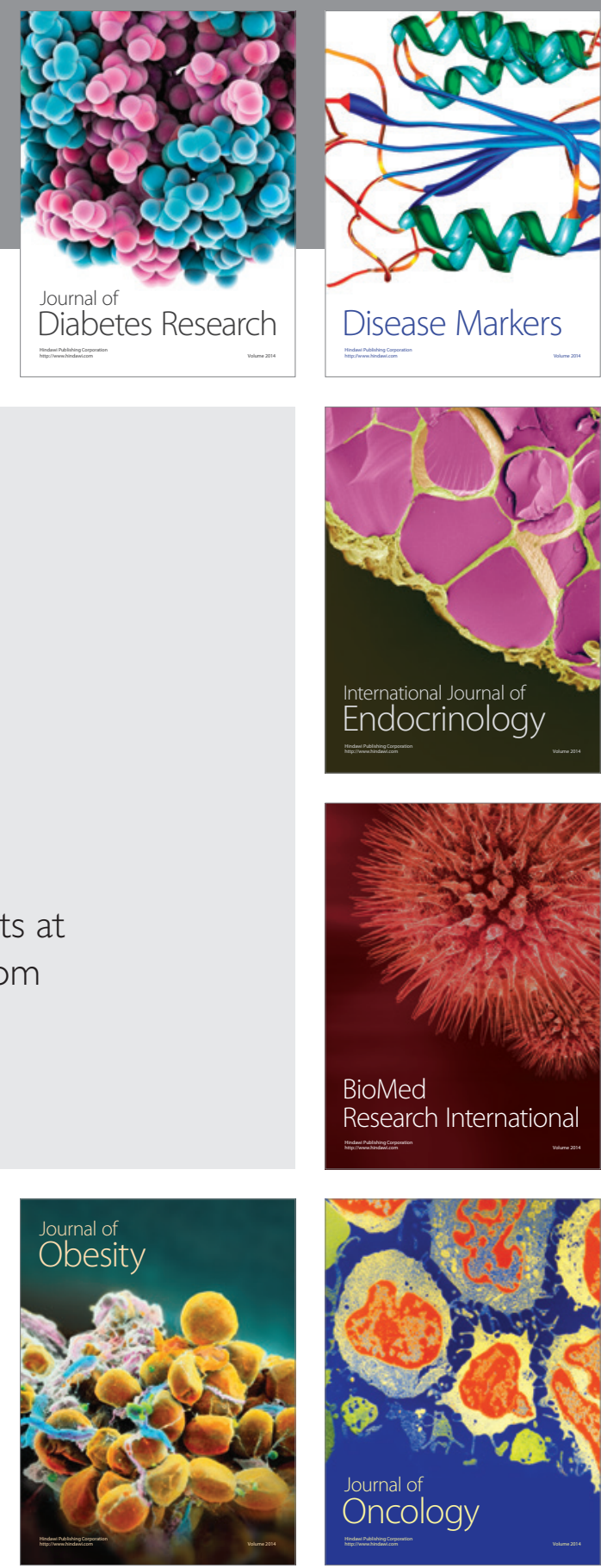

Disease Markers
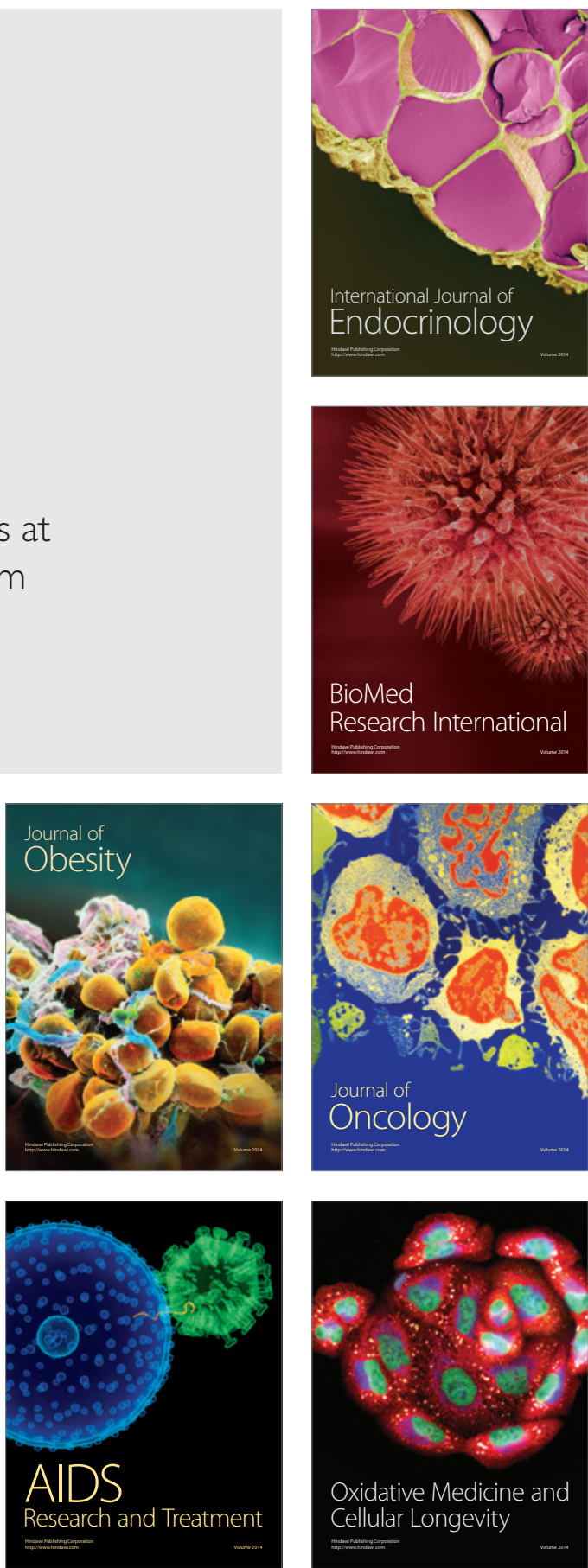